\title{
Brushed nasal epithelial cells are a surrogate for bronchial epithelial CFTR studies
}

\author{
John J. Brewington, ${ }^{1,2}$ Erin T. Filbrandt, ${ }^{1}$ F.J. LaRosa III, ${ }^{1}$ Jessica D. Moncivaiz, ${ }^{1}$ Alicia J. Ostmann, ${ }^{1}$ \\ Lauren M. Strecker, ${ }^{1}$ and John P. Clancy,2 \\ 'Division of Pulmonary Medicine, Cincinnati Children's Hospital Medical Center, Cincinnati Ohio, USA. \\ ${ }^{2}$ Department of Pediatrics, University of Cincinnati College of Medicine, Cincinnati Ohio, USA.
}

\begin{abstract}
Recent advances in the management of cystic fibrosis (CF) target underlying defects in the CF transmembrane conductance regulator (CFTR) protein, but efficacy analyses remain limited to specific genotype-based subgroups. Patient-derived model systems may therefore aid in expanding access to these drugs. Brushed human nasal epithelial cells (HNEs) are an attractive tissue source, but it remains unclear how faithfully they recapitulate human bronchial epithelial cell (HBE) CFTR activity. We examined this gap using paired, brushed HNE/HBE samples from pediatric CF subjects with a wide variety of CFTR mutations cultured at the air-liquid interface. Growth and structural characteristics for the two cell types were similar, including differentiation into mature respiratory epithelia. In electrophysiologic analysis, no correlation was identified between nasal and bronchial cultures in baseline resistance or epithelial sodium channel (ENaC) activity. Conversely, robust correlation was demonstrated between nasal and bronchial cultures in both stimulated and inhibited CFTR activity. There was close correlation in modulator-induced change in CFTR activity, and CFTR activity in both cell types correlated with in vivo sweat chloride measurements. These data confirm that brushed HNE cell cultures recapitulate the functional CFTR characteristics of HBEs with fidelity and are therefore an appropriate noninvasive HBE surrogate for individualized CFTR analysis.
\end{abstract}

Conflict of interest: The authors have declared that no conflict of interest exists.

Submitted: December 19, 2017

Accepted: May 29, 2018

Published: July 12, 2018

Reference information: JCI Insight. 2018;3(13): e99385. https://doi.org/10.1172/jci. insight. 99385.

\section{Introduction}

Cystic fibrosis (CF) is the most common fatal genetic disease in persons of Northern European descent, affecting approximately 70,000 people worldwide $(1,2)$. Early recognition of characteristic alterations in salt/ion transport led to discovery of the genetic cause of $\mathrm{CF}$, mutations in the gene encoding the CF transmembrane conductance regulator (CFTR) protein (3, 4). CFTR is a traffic ATPase chloride and bicarbonate channel present in numerous tissues that primarily functions to regulate epithelial ion and fluid homeostasis (5). Loss-of-function mutations in CFTR lead to complex, multisystem disease, with the majority of morbidity and mortality occurring in the respiratory tract (2). In the CF airway, pathologic dehydration of airway surface liquid and loss of ion transport lead to chronic obstruction, infection, inflammation, and ultimately progressive lung function decline (6).

With more than 2,000 described mutations in the CFTR gene, it is readily apparent that different genetic variants drive a variety of protein consequences and therefore require different interventions for recovery of function $(2,7)$. Much of this understanding was derived from heterologous models expressing high levels of normal or mutant CFTR (e.g., Fisher rat thyroid cells) and in studies of primary human bronchial epithelial cells (HBEs) from lung explants obtained at lung transplantation (8-11). Through this work, many common CFTR mutations have been classified based on the protein defect (e.g., improper folding/trafficking, poor channel conductance, altered channel gating, reduced protein synthesis). Most CFTR mutations, however, remain poorly described, with efforts ongoing to fully characterize their protein defects (12).

Small-molecule therapeutics termed "modulators" targeting dysfunctional CFTR have recently been introduced and hold promise for disease modification $(13,14)$. There are currently 3 FDA-approved CFTR modulators, prescribed in a genotype-based fashion. The first, ivacaftor, is approved for a group of 
mutations representing $<10 \%$ of the $\mathrm{CF}$ population that cause gating, conductance, or splicing defects, as well as mutations demonstrating drug response in laboratory studies. Ivacaftor serves as the current benchmark for modulator therapies; when tested in subjects with G551D CFTR (gating defect), ivacaftor produces $\sim 10 \%$ improvement in lung function and slows the rate of lung function decline $(13,15,16)$. The second available modulator, lumacaftor, is approved in combination with ivacaftor for patients homozygous for the most common CFTR mutation, F508del (trafficking defect). This combination produced smaller improvements in lung function $(\sim 3 \%)$ and a significant reduction in pulmonary exacerbation frequency (14). Finally, a third drug, tezacaftor, was recently approved by the FDA in conjunction with ivacaftor. This combination is approved as an alternative to lumacaftor/ivacaftor for patients homozygous for F508del CFTR, with similar clinical efficacy, as well as for subjects with at least one copy of a tezacaftor/ivacaftor-responsive mutation $(17,18)$.

While genotype-directed therapy represents a form of personalized medicine, it remains imperfect. For those harboring CFTR mutations for which there exists an FDA-approved modulator therapy, there remains a wide range of response, suggesting future opportunities for personalized optimization $(14,16)$. Some of this variability represents patient-specific differences in mutant CFTR activity, as previously reported in HBEs from different donors with common genotypes $(19,20)$. In addition, those with less-common or poorly described mutations are currently excluded from potentially beneficial therapies due to a lack of sufficient numbers for traditional clinical trials. Both problems may be overcome through personalized preclinical model systems, wherein patient tissues are used to generate laboratory models predicting modulator response to drive precision medicine (21).

Established model systems derived from CF patients to test CFTR modulator responsiveness include explant HBEs derived at the time of transplant and intestinal organoids isolated from rectal tissue (11, 2226). The former are not feasible for individualized testing, and the latter require rectal biopsy and specialized techniques for cell isolation, propagation, and CFTR functional analysis. There is a need, therefore, to generate individualized, portable, and noninvasive model systems able to examine the cellular responses to current and emerging CFTR modulators.

In search of an easily obtained, noninvasive source of respiratory tissue, investigators have begun to examine brushed human nasal epithelial cells (bHNEs) (27-30). The nasopharynx harbors a ciliated, pseudostratified respiratory epithelium with many similarities to the bronchial epithelium, making this a feasible model representative of the lower airway. This principle underlies the use of nasal potential difference to detect CFTR function, a well-described CF diagnostic test (31). Early ex vivo studies in surgically obtained polyp specimens confirmed robust CFTR expression that is readily measurable (32). More recently, it has been demonstrated that bHNEs are easily and safely acquired in a nonsurgical fashion through cytologic brush or curettage in individuals ranging from infants to adults (33). Propagation of bHNEs in culture was initially limited by senescence and squamous transformation, which can be mitigated through use of recently developed conditional reprogramming culture (CRC) techniques (34). This method aids in preservation of basal cells and propagation over multiple passages, allowing a single sample to produce multiple differentiated cultures (35).

While it appears logical to pursue bHNEs as a model of CFTR function, it remains unclear how faithfully these cells recapitulate HBE characteristics, including electrophysiologic behavior, expression of respiratory epithelial markers, and response to CFTR modulation. In the current study, we examined whether bHNEs and brushed HBEs (bHBEs) obtained from the same subject exhibited similar characteristics relevant to CFTR functional modulation ex vivo.

\section{Results}

Subject characteristics. Eleven pairs of bHNE and bHBE samples were expanded and tested from 10 subjects, all carrying a clinical diagnosis of CF with a wide range of disease severity. No subjects were related, and no subjects were taking CFTR modulator therapies at the time of sample collection. CFTR genotype was determined by chart review and prior clinical assays, which varied by patient; additional genetic sequencing was not performed, resulting in 4 unidentified alleles across the 10 -subject cohort (subjects $\mathrm{D}, \mathrm{H}$, and $\mathrm{J}$ ). Clinical characteristics of the studied cohort, including CFTR genotype, are summarized in Table 1, which also includes a key to identify each subject throughout the subsequent text and figures.

Growth characteristics and culture success. In all bHNE $(n=102)$ and bHBE $(n=22)$ cultures at our institution over a 2-year period, there was a smaller initial cell number in bHNEs $\left(0.36 \times 10^{6} \pm 0.04 \times 10^{6}\right.$ cells $)$ 
Table 1. Clinical characteristics of brushed human nasal and bronchial epithelial (bHNE and bHBE) cell donors

\begin{tabular}{|c|c|c|c|c|c|c|}
\hline Subject & Age (yr) & Sex & CFTR genotype & $\mathrm{SC}$ (mmol/l) & ppFEV $_{1}$ & Microbiology \\
\hline A & 16 & $M$ & F508del/I507del & 110 & 106 & BM, PA \\
\hline B & 5 & $\mathrm{~F}$ & F508del/R1066C & 101 & - & HF \\
\hline $\mathrm{D}$ & 10 & M & G542X/unidentified & 73 & 110 & MSSA \\
\hline E & 5 & M & F508del/F508del & 119 & 82 & MSSA \\
\hline $\mathrm{F}$ & 4 & $\mathrm{~F}$ & E1371X/2789+2insA & 101 & - & PA \\
\hline 1 & 18 & M & F508del/5T/12TG & 67 & 109 & MSSA \\
\hline J & 10 & $\mathrm{~F}$ & G542X/unidentified & 65 & 83 & PA \\
\hline Average (SEM) & $10.0(1.5)$ & $N / A$ & $N / A$ & $90.8(6.1)$ & $85.9(8.7)$ & $\mathrm{N} / \mathrm{A}$ \\
\hline
\end{tabular}

All 10 donors are represented by letters, which correspond to data points throughout the text and figures. Samples were obtained from subject J twice. Two subjects ( $B$ and $F$ ) were unable to complete spirometry due to age. SC, sweat chloride, taken as the average of both arms; ppFEV, percent predicted forced expiratory volume in 1 second; taken as the highest value in the 6 months preceding sample collection. BM, Burkholderia multivorans; PA, Pseudomonas aeruginosa; HF, Haemophilus influenzae; MSSA, methicillin-sensitive Staphylococcus aureus.

compared with bHBEs $\left(0.69 \times 10^{6} \pm 0.15 \times 10^{6}\right.$ cells; $P=0.003$, Figure $\left.1 \mathrm{~A}\right)$. Despite this, total cell expansion between bHNE and bHBE cultures was equivalent (Figure $1 \mathrm{~B}$ ), as was the amount of time required for cells to achieve confluence for each subsequent passage (Figure 1C) up to passage 4.

Culture success (defined as air-liquid interface [ALI] insert cultures achieving baseline transepithelial resistance $>50 \Omega^{*} \mathrm{~cm}^{2}$ and demonstrating measurable amiloride- and forskolin/IBMX-induced [forskolin/ 3-isobutyl-1-methylxanthine-induced] currents) was achieved in 59\% of bHNE and $73 \%$ of bHBE samples (all CF samples; subsequent experiments on bHNE samples in our laboratory showed improved culture success rate of $67 \%$ [data not shown]). The primary reason for failure was bacterial or fungal contamination during the expansion phase of culture.

Structural characteristics. Immunofluorescence of bHNE and bHBE samples demonstrated key characteristics of mature respiratory epithelia, including a pseudostratified columnar structure. Ciliated cells (acetylated $\alpha$-tubulin), mucus-secreting cells (muc5AC), adherens junctions (E-cadherin), and CFTR were all detected in both monolayer types. Example images from subject $\mathrm{J}$ are provided in Figure 2, and additional images from subjects B and $\mathrm{F}$ are provided in Supplemental Figure 1; supplemental material available online with this article; https://doi.org/10.1172/jci.insight.99385DS1.

Ion transport. ALI cultures were mounted in Ussing chambers and studied under voltage-clamp conditions in asymmetric chloride solutions (see Methods). Baseline resistance was variable across samples and donors, with statistically significant differences observed within 4 paired samples (Figure 3A). This resulted in an aggregate trend toward increased resistance in bHBE samples that was not significant (Figure 3B; $P=$ 0.97). ENaC activity, as measured by amiloride-inhibited current change, was also variable; there were statistically significant differences within pairs from 5 donors (Figure 3C). Based on group comparisons, there was no difference in ENaC activity between bHNEs and bHBEs (Figure 3D; $P=0.74$ ). There was no significant correlation of bHNE and $\mathrm{bHBE}$ baseline resistance or ENaC activity (Figure 3, E and F, respectively). Bland-Altman analyses revealed a bias toward larger baseline resistance measurements $\left(+67.3 \Omega^{*} \mathrm{~cm}^{2}\right)$ in bHBE cultures, though the line of no difference fell within the $95 \% \mathrm{CI}$ of this mean (Supplemental Figure 2, A and B). Conversely, there was a bias toward more negative (i.e., larger absolute value) amiloride-sensitive current measurements in bHNEs $\left(-13.7 \mu \mathrm{A} / \mathrm{cm}^{2}\right)$, with the line of no difference falling within the $95 \%$ CI of the mean (Supplemental Figure 2, C and D).

CFTR activity was analyzed in two fashions; first through stimulated currents with forskolin/ IBMX and VX-770 (ivacaftor) (following ENaC inhibition with amiloride), and then inhibited currents with CFTR Inhibitor-172 (CFTR Inh172). Values of stimulated CFTR short-circuit current $\left(I_{s c}\right)$ ranged from almost no function (subject A bHBEs, $0.1 \mu \mathrm{A} / \mathrm{cm}^{2}$ ) to WT CFTR function (subject J2 bHNEs, $38.5 \mu \mathrm{A} / \mathrm{cm}^{2}$ ), with similar CFTR Inh172 $I_{s c}$ (Figure 4A). Stimulated CFTR currents were statistically different within 4 paired samples, as were inhibited CFTR currents. As a group, there was 


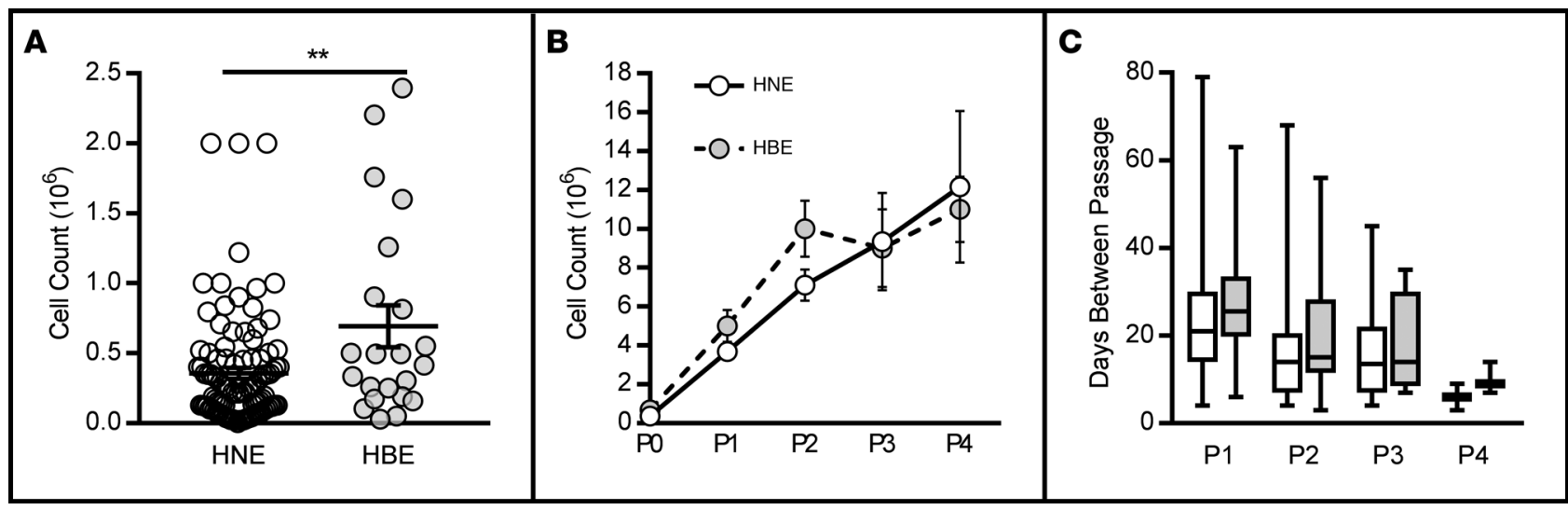

Figure 1. Center-specific growth characteristics of brushed human nasal and bronchial epithelial cells (bHNEs and bHBEs). (A) Initial sample cell count for bHNEs (white circles, $n=102$ ) and bHBEs (gray circles, $n=22$ ). ${ }^{* *} P<0.01$ by 2 -tailed $t$ test; bars represent mean \pm SEM. (B) Cumulative cell growth. Note that the passage $\mathbf{O}(\mathrm{PO})$ time point reflects the initial cell counts shown in $\mathbf{A}$; error bars indicate SEM. (C) Time between each passage, with no significant difference between bHNE (white bars) and bHBE (gray bars) cultures at any passage. Boxes represent median (center line), 25th and 75th quartiles (box limits), and minimum/maximum (whiskers) values. Note that in $\mathbf{B}$ and $\mathbf{C}$, the number of ongoing cultures in each tissue type dwindles with advancing passage (P1: 87/22; P2: 32/15; P3: 13/3, P4: 3/3 for bHNE/bHBE, respectively) due both to cell-specific (e.g., contamination) and protocol-specific (e.g., systematic banking at P2) factors. Using multiple $t$ tests (with Holm-Šidák correction), no significant difference was noted between bHNE and bHBE total cell count (B) or time to passage (C) at any passage beyond PO.

no difference in stimulated $(P=0.56)$ or inhibited $(P=0.11)$ CFTR currents between $\mathrm{bHNE}$ and $\mathrm{bHBE}$ samples (Figure 4B). There was a statistically significant correlation of bHNE and bHBE pairs for both stimulated (Figure 4C; $P=0.001, R^{2}=0.761$ ) and inhibited (Figure $4 \mathrm{D} ; P=0.001, R^{2}=0.743$ ) CFTR currents by linear regression. Bland-Altman analyses revealed a bias toward larger stimulated CFTR $I_{s c}$ measurements $\left(+1.5 \mu \mathrm{A} / \mathrm{cm}^{2}\right)$ in bHBE cultures, with the line of no difference within the $95 \% \mathrm{CI}$ of this mean (Supplemental Figure 3, A and B). Similarly, there was a bias toward more negative (i.e., larger absolute values) Inh712-sensitive current measurements in bHBEs $\left(-2.6 \mu \mathrm{A} / \mathrm{cm}^{2}\right)$, with the line of no difference falling within the $95 \%$ CI of the mean (Supplemental Figure 3, C and D).

Detection of CFTR modulation. Select inserts from all paired samples, regardless of mutation, were pretreated with $3 \mu \mathrm{M}$ VX-809 (lumacaftor) for 48-72 hours prior to analysis. VX-809 incubation did not change baseline resistance in bHNEs (control, 290.4 vs. VX-809, $294.3 \Omega^{*} \mathrm{~cm}^{2} ; P=0.97$ ) or bHBEs (control, 351.2 vs. VX-809, $378.5 \Omega^{*} \mathrm{~cm}^{2} ; P=0.81$ ). Incubation with VX-809 produced varying changes in ENaC activity that were donor dependent. These ranged from a near-complete loss of amiloride-inhibited currents (subject $\mathrm{J})$ to more than a doubling (subject $\mathrm{G}$ ). There was no statistical correlation in absolute VX-809-induced change in $\mathrm{ENaC}$ activity in paired nasal and bronchial cells by linear regression $\left(P=0.057, R^{2}=0.381\right.$; Figure $5 \mathrm{~A})$. Bland-Altman analyses revealed a bias toward more positive absolute VX-809-induced changes in amiloride-inhibited currents $\left(+4.7 \mu \mathrm{A} / \mathrm{cm}^{2}\right)$ in bHBE cultures, with the line of no difference within the $95 \%$ CI of this mean (Supplemental Figure 4, A and B). When analyzed as a relative VX-809-induced change (compared with absolute $I_{s c}$ values), there was no correlation in percent change in ENaC activity from baseline in paired nasal and bronchial cells by linear regression $\left(P=0.063, R^{2}=0.368\right.$; Supplemental Figure 5A). Bland-Altman analysis demonstrated a small bias toward more positive relative VX-809-induced changes in amiloride-sensitive currents $\left(+12.1 \mu \mathrm{A} / \mathrm{cm}^{2}\right)$, with the line of no difference falling within the $95 \% \mathrm{CI}$ of the mean (Supplemental Figure 6, A and B).

VX-809 incubation produced variable changes in both stimulated (forskolin/IBMX and VX-770) and inhibited (Inh172) CFTR currents that were donor- and CFTR mutation-dependent. These ranged from significant reductions (subject $\mathrm{J}$ ) to $>200 \%$ improvements in both stimulated and inhibited currents (subject A). The absolute change in CFTR currents in paired bHNEs and bHBEs induced by VX-809 exposure correlated strongly by linear regression for both stimulated $\left(P<0.0001, R^{2}=0.909\right.$; Figure $\left.5 \mathrm{~B}\right)$ and inhibited $\left(P<0.0001, R^{2}=0.934\right.$; Figure $5 \mathrm{C}$ ) currents. Minimal bias toward more positive changes in bHBE samples for both stimulated and inhibited CFTR current was demonstrated upon Bland-Altman analysis (Supplemental Figure 4, C-F), with the line of no difference falling within the $95 \%$ CI for these means. When analyzed as a relative VX-809-induced change, the percent change in CFTR function from baseline correlated well within subjects for both stimulated 


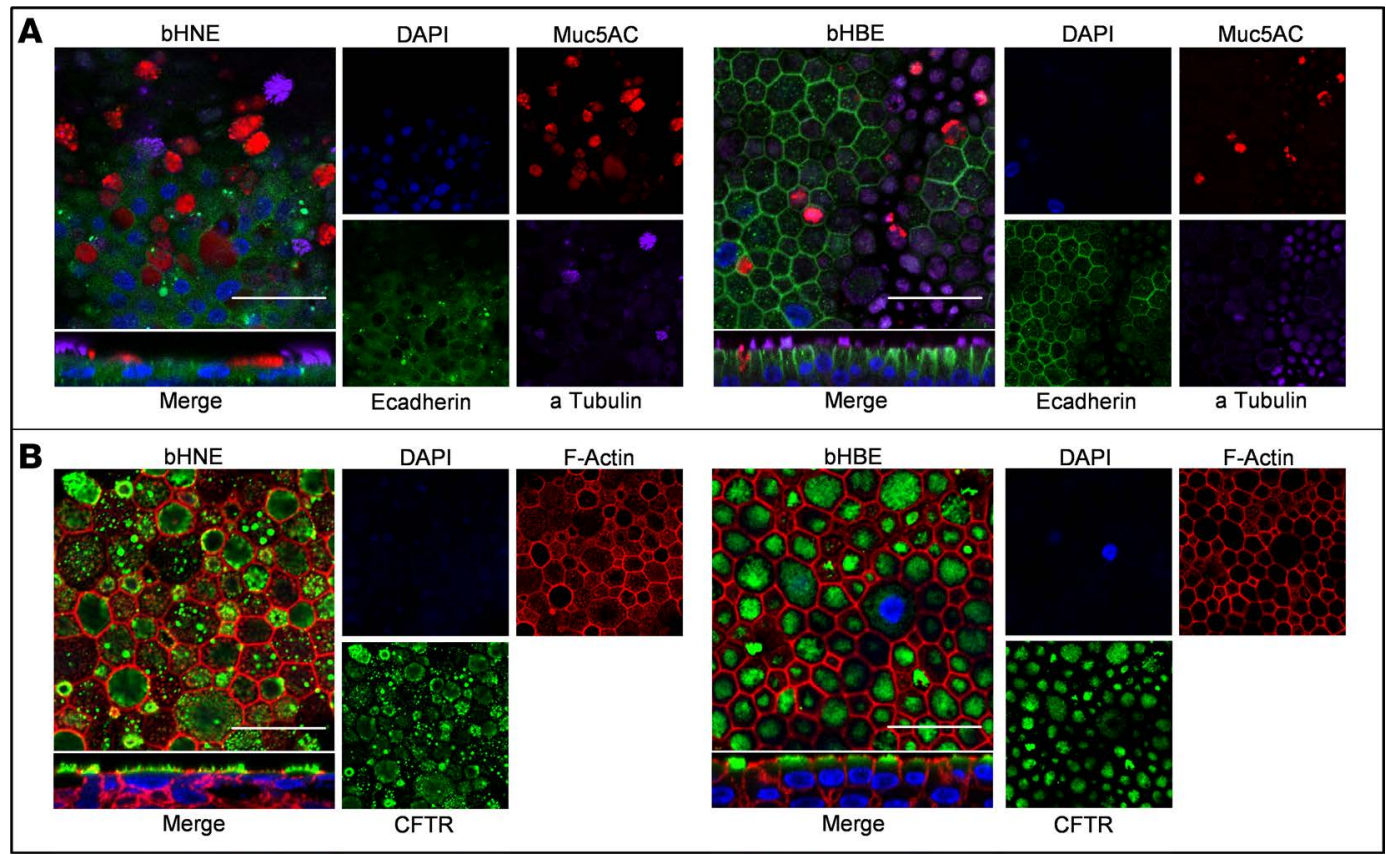

Figure 2. Brushed human nasal and bronchial epithelial cells (bHNEs and bHBEs) share similar characteristics of a mature respiratory epithelium. (A) Detection of adherens junctions (E-cadherin, green), mucus-producing cells (muc5AC, red), and ciliated cells (acetylated $\alpha$-tubulin, purple) in both bHNE (left) and bHBE (right) samples. (B) Similar CFTR (green) detection between paired bHNE (left) and bHBE (right) samples. All samples were taken from subject J (Table 1) and are representative of other subjects (3 paired samples; see Supplemental Figure 2 for additional subject images), though CFTR staining varied with mutation. Scale bars: $25 \mu \mathrm{m}$

$\left(P=0.0004, R^{2}=0.808 ;\right.$ Supplemental Figure 5B) and inhibited $\left(P<0.0001, R^{2}=0.909 ;\right.$ Supplemental Figure $5 C)$ currents. Similar to analysis of the absolute change in CFTR function, Bland-Altman analysis demonstrated minor bias toward larger stimulated and inhibited relative CFTR function changes in bHBE samples, with the line of no difference falling within the $95 \% \mathrm{CI}$ of these mean values (Supplemental Figure 6, C-F).

Correlation with sweat chloride. Stimulated CFTR-dependent $I_{s c}$ (forskolin/IBMX and VX-770, no VX-809 pretreatment) for both bHNEs and bHBEs was plotted against subject baseline (non-modulator-treated) sweat chloride (SC) and compared; for the single subject sampled twice, the $I_{s c}$ values were averaged. For both tissue types, there was a strong correlation with $\mathrm{SC}$ by nonlinear regression, with similar regression characteristics (bHNEs, $P=0.0008, R^{2}=0.764$; bHBEs, $P<0.0001, R^{2}=0.866$ ), as shown in Figure 6 . These regression curves demonstrated relatively low $I_{s c}$ responses in both cell types above an SC of 90 , quickly rising in an exponential fashion as SC fell into the 60s. These curves suggest a highly dynamic range of functional CFTR detection in bHNEs and bHBEs for subjects with baseline SC values between 60 and 90 .

\section{Discussion}

Primary HBEs are an established model system to examine CF disease pathology and an important tool in CFTR modulator development. In this article, we present a number of key similarities between same-subject pediatric $\mathrm{CF}$ bHNEs and bHBEs grown at ALI, supporting the concept of bHNE-derived, personalized models for CFTR-focused investigation. This work differs from past studies in several ways. Previous functional characterization of nasal and bronchial epithelia focused on aggregate analysis, whereas the work reported herein evaluated paired, same-subject samples (29). Additionally, most work to date has compared bHNEs with HBEs harvested from lung explants, a substantially different method. We sought to minimize bias in our comparisons by using brushed cells from the upper and lower respiratory tract that were processed, expanded, differentiated, and studied with equivalent protocols in parallel and by the same staff. Finally, this work focused on pediatric subjects and describes results from a wide range of $\mathrm{CF}$ mutations, spanning near-WT CFTR to severe CFTR dysfunction.

In our ALI-based culture models, we noted a number of key similarities and differences between tissue types. Growth characteristics were equivalent, despite a larger initial sample in bHBEs (Figure 1); even this 


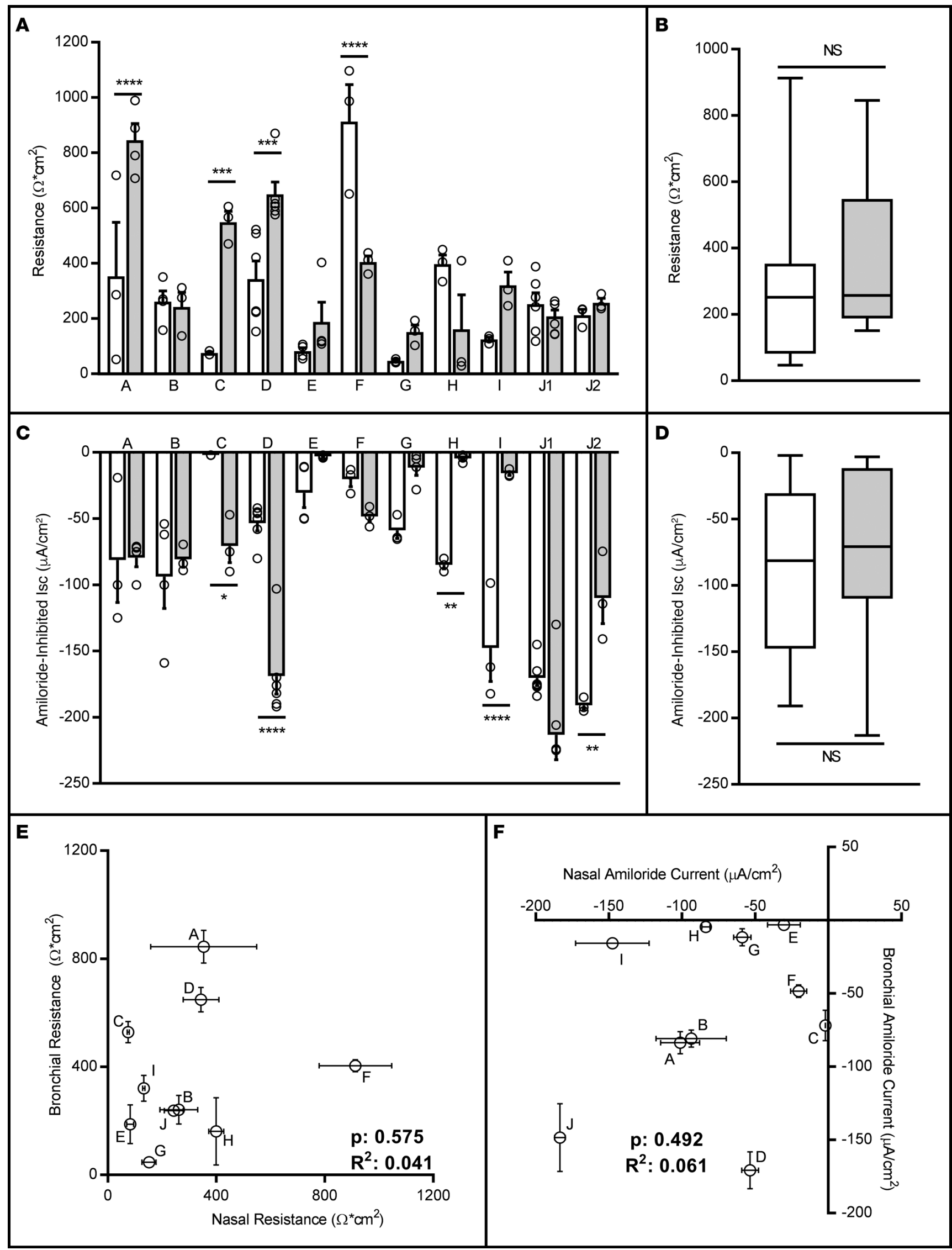


Figure 3. There is poor correlation between brushed human nasal epithelial cells (bHNEs and bHBEs) for baseline resistance and epithelial sodium channel (ENaC) activity. Baseline resistance values for bHNE (white bars) and bHBE (gray bars) samples for each subject (A) and aggregate data for all donors (B). ENaC activity (as measured by amiloride-inhibited short-circuit current $\left[I_{s c}\right]$ ) in bHNE (white bars) and bHBE (gray bars) samples for each subject (C) and aggregate data for all donors (D). (E) Baseline resistance in bHNE ( $x$ axis) and bHBE ( $y$ axis) is plotted for each subject; linear regression was nonsignificant. (F) ENaC activity is similarly plotted, with no significant correlation. $n=3-6$ inserts per sample and condition; individual replicates are noted by circles in $\mathbf{A}$ and $\mathbf{C}$ to demonstrate distribution. ${ }^{*} P<0.05,{ }^{* *} P<0.01,{ }^{* * *} P<0.001,{ }^{* * * *} P<0.0001$. Two-way ANOVA with Tukey's correction for multiple comparisons was used for $\mathbf{A}$ and $\mathbf{C}$, while unpaired, 2-sided $t$ tests were used for $\mathbf{B}$ and $\mathbf{D}$. Individual sample labels correlate with donor codes shown in Table 1. Error bars in A, C, E, and $\mathbf{F}$ represent SEM. Boxes in $\mathbf{B}$ and $\mathbf{D}$ represent median (center line), 25th and 75th quartiles (box limits), and minimum/maximum (whiskers) values.

difference may be related to the smaller number of bHBE samples and related skewing of that data pool due to outliers. Significant variability was noted in both cell types in the time to confluence in passages 1 and 2 (Figure 1C); this variability was primarily related to initial sample size (with larger samples reaching confluence faster). The variance declined with advancing passage due to the laboratory practice of banking samples once growth slowed, thereby selecting for more rapidly growing samples to reach passages 3 and 4. Notably, we observed a higher contamination rate in bHNEs, though this gap has closed with advancing experience (at our center in the 6 months prior to writing of this manuscript, bHNE success was at $67 \%$, while bHBE success remained at $73 \%$ for CF cultures). Improved contamination rates have followed centralization of providers procuring samples and technicians preparing media, as well as adoption of the described antimicrobial media use for all cultures (Table 2). Any difference in bHNE contamination rates versus those of bHBEs, however, was offset by the ease of bHNE collection, and indeed repeated collection if necessary, in the non-sedated patient.

Both tissue types expressed important structural characteristics and proteins consistent with classical respiratory epithelia (Figure 2 and Supplemental Figure 1). The pseudostratified columnar phenotype seen in bHBEs was generally recapitulated in bHNEs; previous studies have also demonstrated pseudostratified columnar epithelia in bHNE cultures (29). Despite minor differences, both bHNE and bHBE samples expressed key markers of a mature respiratory epithelium, including ciliated cells, mucus-producing cells, adherens junctions, apical versus basolateral polarization, and CFTR expression. The differences noted between cell types were not consistent (e.g., between donors), as demonstrated in comparisons of the 3 donor pairs in Figure 2 and Supplemental Figure 1. Prior work has used electron microscopy to demonstrate similar characteristics in pediatric CF bHNE cultures, confirming these results (36).

There was poor within-subject correlation between bHNE and bHBE monolayers for transepithelial resistance, which likely represents cell sampling variability or variance in monolayer maturation over time (Figure 3). While there was no difference in $\mathrm{ENaC}$ activity between bHNEs and bHBEs in aggregate, there was also no within-subject correlation across the samples (Figure 3). Variance in ENaC activity between bHNE and bHBE samples appeared random in Bland-Altman analyses, with a slight bias toward more negative measurements in bHNE samples (though the line of no difference consistently fell within the $95 \% \mathrm{CI}$ of the mean). Ultimately, these data imply variability in the individual monolayers, as opposed to inherent tissue differences in $\mathrm{ENaC}$ activity, but further study will be required for confirmation. Nonetheless, these results do raise questions regarding the suitability of bHNEs to replicate bHBE behavior for studies of $\mathrm{ENaC}$.

The primary focus of this study was on electrophysiologic assay of CFTR function, which correlated well within subjects for nasal and bronchial cells. Values of stimulated CFTR function in bHNEs were similar or higher than those previously reported for CF subjects, likely reflecting the wide range of CFTR function in this diverse study group, though technical items (such as media composition) in the culture process may also drive these differences (29). In our paired analyses, both stimulated and inhibited CFTR-dependent currents correlated well, supporting the use of bHNEs as a proxy for bHBEs (Figure 4, C and D). There was a trend toward larger stimulated and inhibited CFTR-dependent currents in bHBEs, though this did not reach statistical significance. This bias toward larger CFTR currents in bHBEs was again demonstrated in Bland-Altman analyses (Supplemental Figure 3), with an average increase in bHBE stimulated CFTR $I_{s c}$ of $1.5 \mu \mathrm{A} / \mathrm{cm}^{2}$, and an increase in the absolute value of Inh172-sensitive current of $2.6 \mu \mathrm{A} / \mathrm{cm}^{2}$. Both of these average differences, however, were nonsignificant, as the $95 \%$ CI of the mean bias lines contained the line of no difference.

Critically, CFTR modulation with VX-809 produced detectable changes in CFTR function in both tissues across a variety of CFTR mutations, even in the subjects with the smallest baseline currents. This includes functional improvement in 3 subjects (2 F508del homozygotes [subjects $\mathrm{C}$ and $\mathrm{E}$ ] and 1 with a 


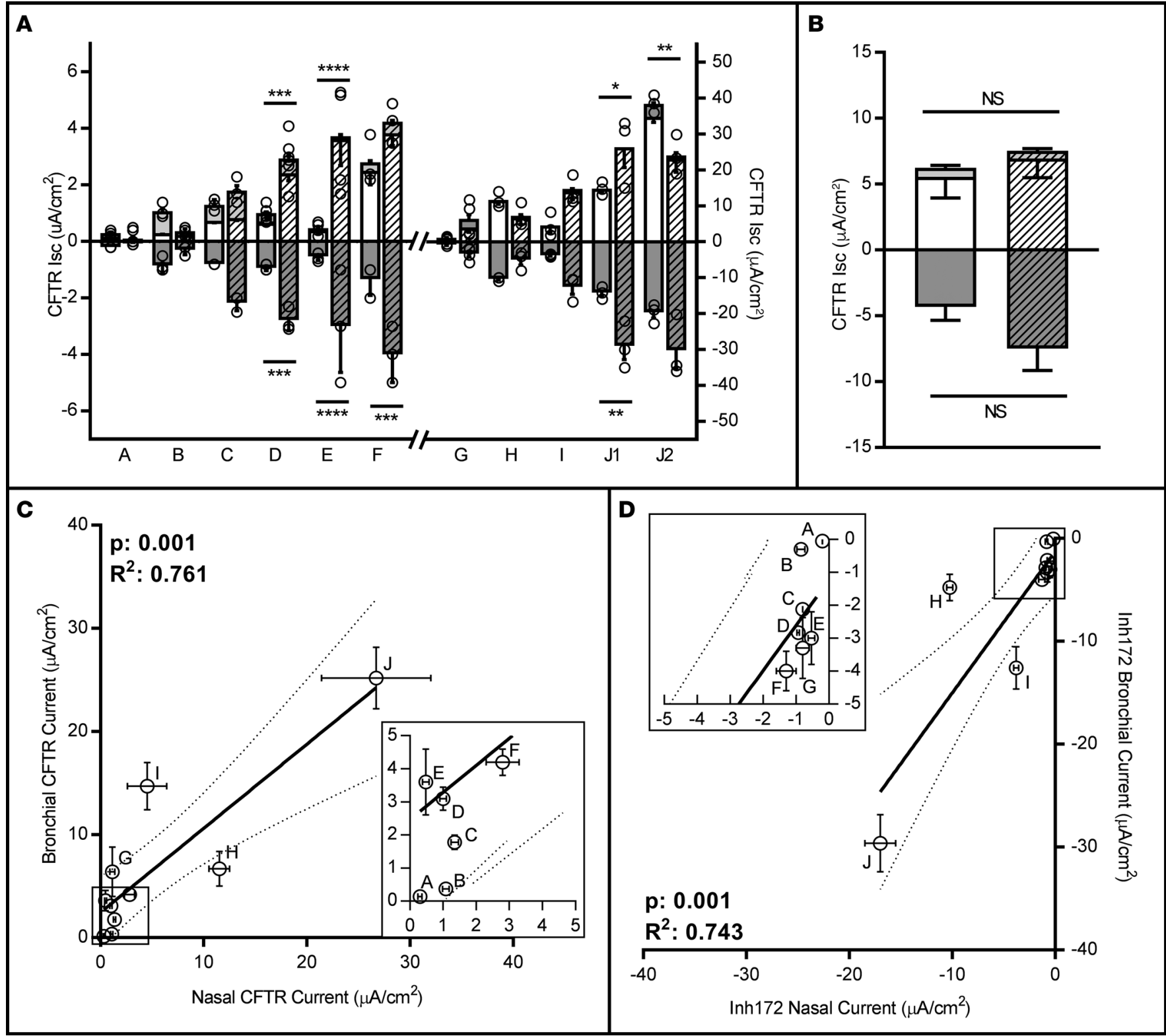

Figure 4. Stimulated and inhibited CFTR activity correlate well between brushed human nasal and bronchial epithelial cells (bHNEs and bHBEs). (A) Both stimulated (forskolin/IBMX, white; VX-770, light gray) and inhibited (Inh172, dark gray) CFTR-dependent short-circuit current (Isc) for paired bHNE (solid bars) and bHBE (hatched bars) samples for all subjects. (Note the different $y$-axis scales used for subjects A-F to the left and subjects G-J to the right.) (B) Aggregate data for all donors. (C) Total stimulated CFTR current (forskolin/IBMX+VX-770) in bHNEs ( $x$ axis) and bHBEs ( $y$ axis) are plotted for each subject; linear regression with $95 \% \mathrm{CI}$ (dotted lines) is shown. (D) Inhibited CFTR currents (Inh172) are similarly plotted; linear regression with $95 \%$ $\mathrm{Cl}$ (dotted lines) is shown. $n=3-6$ inserts per sample and condition; individual replicates are noted by circles in $\mathbf{A}$ (stimulated: forskolin/IBMX+VX-770) to demonstrate distribution. ${ }^{*} P<0.05,{ }^{* *} P<0.01$, ${ }^{* *} P<0.001,{ }^{* * * *} P<0.0001$. Two-way ANOVA with Tukey's correction for multiple comparisons was used to compare stimulated (forskolin/IBMX+VX-770) and inhibited (Inh172) CFTR currents in A, while unpaired, 2-sided $t$ test was used for the same comparison in B. Individual sample labels correlate with donor codes shown in Table 1. Error bars in A, C, and $\mathbf{D}$ represent SEM. Boxes in B represent median (center line), 25th and 75th quartiles (box limits), and minimum/maximum (whiskers) values.

single F508del mutation and a second folding mutation, I507del [subject A]) and severe functional decline in both cell types from both samples obtained from a subject with near-WT CFTR function (G542X/ unknown CFTR mutation, subject J). VX-809-induced changes in both stimulated and inhibited CFTR function correlated well across this diverse cohort, whether assessed as an absolute change in $I_{s c}$ (Figure 5, B and C) or as change relative to the baseline (Supplemental Figure 5, B and C). Similarly, Bland-Altman analyses demonstrated minimal bias in both absolute and relative VX-809-induced changes in CFTR activity, and the demonstrated CIs of this bias consistently included the line of no difference. Thus, these 

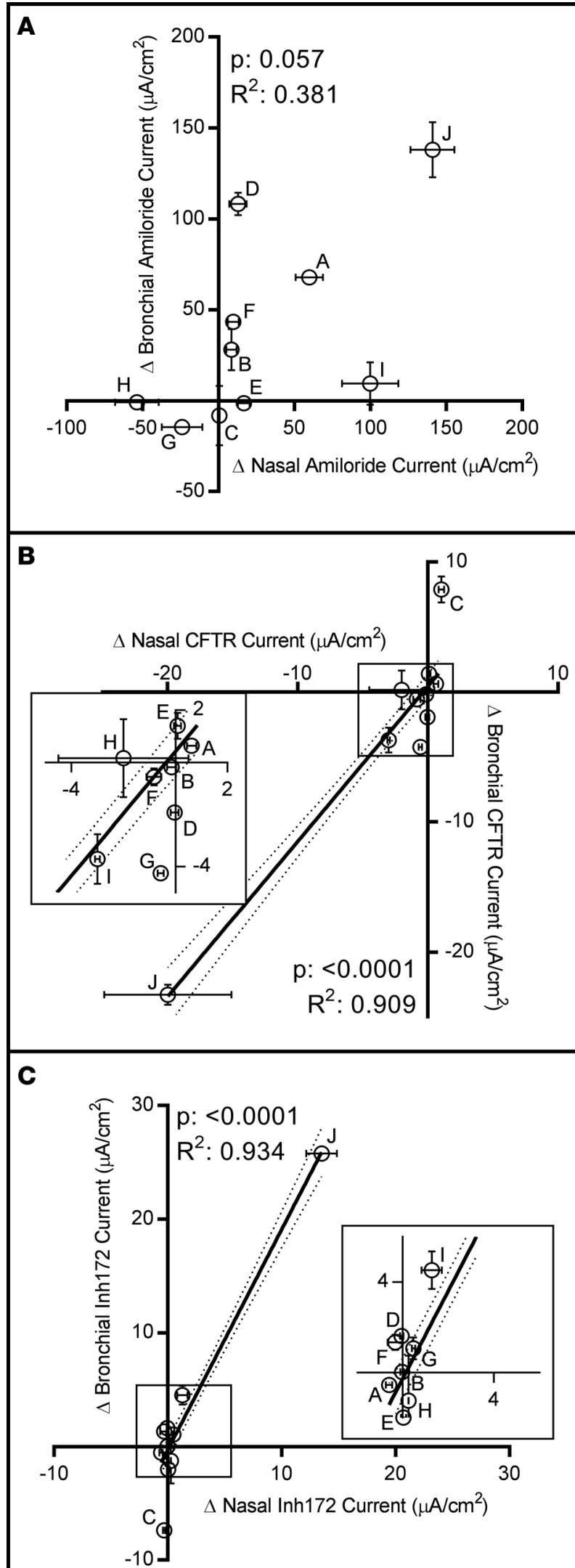

Figure 5. Absolute VX-809-induced changes in CFTR currents correlate well for same-subject brushed human nasal and bronchial epithelial (bHNE and bHBE) samples. (A) Absolute change in amiloride-inhibited currents in VX-809-pretreated bHNE ( $x$ axis) and bHBE ( $y$ axis) paired samples; linear regression was nonsignificant. Absolute $V X-809$-induced change in stimulated (forskolin/IBMX+VX-770) (B) and inhibited (Inh172) (C) CFTR currents for paired bHNE ( $x$ axis) and bHBE ( $y$ axis) samples, along with linear regression characteristics with $95 \% \mathrm{Cl}$ (dotted lines). $n=3-6$ inserts per sample and condition. Individual sample labels correlate with donor codes shown in Table 1. Error bars represent SEM.

data confirm strong correlation, though not exact matching, of pharmacologic CFTR modulation between paired bHNE and bHBE samples.

As noted in Figure 5, VX-809 induced a negative change in CFTR-dependent $I_{s c}$ in several subjects, ranging from very mild reduction (e.g., subject I) to almost complete loss of CFTR function (subject $\mathrm{J}$, with consistent results from 2 separate sample sets). Impairment of CFTR activity following VX-809 exposure has been previously reported, though not extensively studied (29). It is possible that this reflects a toxic effect of the drug, or off-target effects; however, the same drug (same stock and batch) yielded improvement or no change in the other subjects, making this less likely. Moreover, baseline (unstimulated, before change to an apical low-chloride solution) electrical resistance was not altered in the VX-809-exposed samples from subject J, suggesting cell toxicity was not involved in the negative CFTR effects. As VX-809 is believed to interact directly with CFTR, it is possible that this interaction can have negative consequences for some CFTR mutations. This possibility has significant implications, as efforts are underway to expand existing modulator drugs to new patient groups. Thus, further study is necessary to determine the mechanism of this non-F508del CFTR impairment with VX-809 and to assess if similar effects occur with other modulators.

Both bHNE and bHBE models were assessed for correlation to SC as an in vivo biomarker of CFTR function. Interestingly, models of both cell types fit a similar nonlinear (log-log) regression line when plotted against SC values (Figure 6). A previous study has suggested a linear association between nasal potential difference and bHNE ALI cultures, but there has not been a direct link to SC reported (29). This nonlinear relationship may simply represent the small sample size in our study, but SC has also been regarded as a nonlinear measure of CFTR function $(37,38)$. The key inflection point of both curves suggests a highly dynamic range of CFTR detection between SC values of approximately 60 and $90 \mathrm{mmol} / 1$, which is also a critical window of pathologic importance. SC values of $>90 \mathrm{mmol} / \mathrm{l}$ are typically associated with minimal CFTR function and a "severe" phenotype. In contrast, SC values that approach $60 \mathrm{mmol} / 1$ are often observed in patients with partial-function CFTR mutations and pancreatic sufficiency. Indeed, $60 \mathrm{mmol} / 1$ is a diagnostic threshold for CF, and highly effective CFTR modulator therapy (e.g., ivacaftor in patients with gating CFTR mutations) can reduce SC values below this threshold $(39,40)$. Thus, ex vivo detection of residual CFTR function in samples from subjects with SC values between 60 and $90 \mathrm{mmol} / 1$ reinforces the fidelity of bHNE and bHBE models to the in vivo condition.

There are several limitations of the present work. Subjects were enrolled in an effort to study a wide range of CFTR function, and all meet clinical criteria for a diagnosis of CF. This approach, however, 


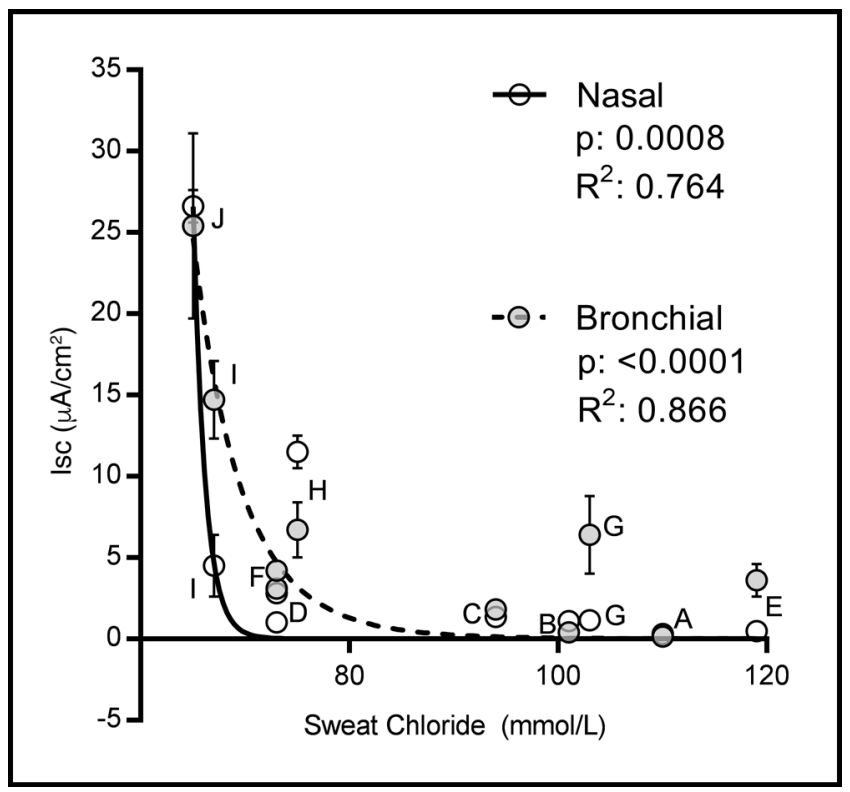

Figure 6. Similar, nonlinear inverse correlation between CFTR activity and sweat chloride (SC) in both brushed human nasal and bronchial epithelial cells (bHNEs and bHBEs). Stimulated CFTR short-circuit current (Isc; forskolin/IBMX+VX-770, $y$ axis) is plotted against SC values ( $x$ axis) for both bHNE (white circles) and bHBE (gray circles) samples. Similar nonlinear (log-log) regression lines are plotted for bHNE (solid line) and bHBE (dotted line) samples; regression values are as indicated. For subject J (2 paired samples), Isc values from repeated samples were averaged. $n=1 \mathrm{SC}$ measurement for each subject, 3-6 inserts per tissue sample per subject. Individual sample labels correlate with donor codes shown in Table 1. Error bars represent SEM.

yields a group that is heterogeneous and not fully representative of the larger CF population (with only 2 subjects homozygous for F508del CFTR). Moreover, many samples demonstrated very small CFTR responses, which may limit the precision of correlation analyses. The interpretation of these analyses is further complicated by inclusion of 4 unidentified CFTR mutations (2 subjects homozygous for G542X and 1 with no identified mutations). While this limits genotype/function comparisons, these subjects were included to cover a wide range of CFTR activity. Importantly, this heterogeneous population represents that in which patient-derived model systems may be of greatest benefit, as they are not routinely part of modulator trials. The small number of subjects and wide variability in CFTR function included in this study also preclude the broad adoption of bHNEs without additional validation, including studies to tie model system readouts to in vivo CFTR activity. Critically, it is unclear what (if any) model system results predict patient response to CFTR modulation (e.g., absolute vs. relative change in CFTR function, etc.). Additional study will be required to vet such models as preclinical predictors of treatment success or failure. Moreover, rigorous study of bHNE models will be necessary to determine whether the variance of the test is beyond its discriminating capacity for pharmacologic CFTR modulation and to establish the repeatability of the assay within a subject.

Table 2. Media composition for cell culture

\begin{tabular}{|c|c|c|}
\hline $0.4 \mu \mathrm{g} / \mathrm{ml}$ hydrocortisone & $0.4 \mu \mathrm{g} / \mathrm{ml}$ hydrocortisone & 20 nM hydrocortisone \\
\hline $5 \mu \mathrm{g} / \mathrm{ml}$ insulin & $5 \mu \mathrm{g} / \mathrm{ml}$ insulin & $2.5 \mu \mathrm{g} / \mathrm{ml}$ insulin \\
\hline $8 \mathrm{ng} / \mathrm{ml}$ cholera toxin & $8 \mathrm{ng} / \mathrm{ml}$ cholera toxin & $0.25 \%$ bovine brain extract \\
\hline 5-10 $\mu \mathrm{M}$ Y-27632 (Rho kinase inhibitor) & 5-10 $\mu \mathrm{M}$ Y-27632 (Rho kinase inhibitor) & $2.5 \mu \mathrm{g} / \mathrm{ml}$ transferrin \\
\hline $100 \mu \mathrm{g} / \mathrm{ml}$ tobramycin & & $250 \mathrm{nM}$ ethanolamine \\
\hline $100 \mu \mathrm{g} / \mathrm{ml}$ ceftazidime & & $1.5 \mu \mathrm{M}$ epinephrine \\
\hline $100 \mu \mathrm{g} / \mathrm{ml}$ vancomycin & & $250 \mathrm{nM}$ phosphoethanolamine \\
\hline $0.5-2.5 \mu \mathrm{g} / \mathrm{ml}$ amphotericin B & & $10 \mathrm{nM}$ retinoic acid \\
\hline
\end{tabular}


Our studies focused primarily on electrophysiologic end points, not other airway epithelial cell-specific outcomes (e.g., transcriptomics, proteomics). It is important to note that bHNE cultures may be an inadequate model of HBEs for other aspects of disease and study, especially for pathology with clinical variance between the upper and lower airways. Previous work has demonstrated similar epithelial structure, ciliary activity, and inflammatory profiles between cell types, while a prior report noted differences in transcriptional signatures between cells derived from the upper and lower airway (41-46). Our focus on electrophysiology allows for careful comparison of CFTR-dependent ion transport but does not assess bicarbonate transport (an important aspect of CFTR function). Slower-growing cultures may be predisposed to changes in ENaC or CFTR expression, confounding results; we offset this possibility by using only cultures of passage 1 (47). Additionally, our studies utilized CRC methodologies, which may alter the native programming of the cells. A previous report indicated that employing these methods in bHNE samples enriched the population for basal cells (35). We sought to control for independent variables induced by CRC by treating bHNEs and bHBEs identically after acquisition, recognizing that subtle differences in culture conditions may optimize performance of nasal versus bronchial monolayers.

In conclusion, we have demonstrated that bHNEs recapitulate many characteristics of bHBEs within subjects by completing careful parallel cell acquisition, expansion, maturation, and analysis. While the results were not identical, there is a strong correlation between CFTR function and quantification of pharmacologic CFTR modulation across the paired cell types. These data support the use of bHNEs as a noninvasive proxy for bHBEs for individualized CFTR study.

\section{Methods}

Subject enrollment. Subjects with documented CF undergoing clinically indicated bronchoscopy procedures were enrolled, and limited clinical data were collected from the electronic medical record, deidentified, and recorded, including baseline SC, lung function, age, sex, CFTR genotype, and respiratory microbiology; all values were obtained before modulator therapy, where applicable. No subjects were on CFTR modulator therapy at the time of sample acquisition. Exclusion criteria included nasal polyposis or airway friability, in which case the clinician deemed performing the cell collection procedure to be unsafe.

Cell procurement, processing, and expansion. bHNEs were obtained through curettage (rhinoprobe, Arlington Scientific Inc.) or cytology brushing (CYB-1, Medical Packaging Corp.) of the inferior turbinate, pooling cells from both nostrils into a single 15-ml conical filled with antibiotic media (see Table 2). bHBEs were obtained by bronchoscopically brushing the bilateral mainstem bronchi under direct endoscopic visualization, pooling cells from both bronchi into a single $15-\mathrm{ml}$ conical filled with antibiotic media. All bronchoscopy procedures were performed with either a BF-XP160F or BF-MP160 pediatric bronchoscope and brushing with a BC-203D-2006 1.2-mm or BC-202D-2010 2.0-mm cytology brush, accordingly (Olympus Corp.).

Cell expansion was performed as previously described, with minor modifications $(28,34,35)$. bHNEs and $\mathrm{bHBEs}$ were kept separate, with mirrored steps performed for each sample. Cells were rinsed off of the curettes/brushes with media, collected, and centrifuged at $360 \mathrm{~g}$ for 5 minutes. The resultant pellets were suspended in Accutase (Innovative Cell Technologies Inc.) and centrifuged at $360 \mathrm{~g}$ for 5 minutes. Cells were subsequently suspended in antibiotic media and seeded into cell culture dishes precoated with VitroCol (Advanced BioMatrix) and growing irradiated mouse embryonic fibroblast feeder cells (GlobalStem). bHNEs and bHBEs were maintained in dishes until confluent, with media changed daily; media was changed from antibiotic media to expansion media (see Table 2) after 5 days.

To passage confluent cell culture dishes, media was removed, and 0.1\% trypsin (Sigma-Aldrich) was added for 5 minutes to facilitate detachment. This mixture was collected and centrifuged at $360 \mathrm{~g}$ for 5 minutes and the supernatant removed. The resultant pellet was then passaged to a new dish or onto permeable inserts for ALI culture. All presented functional experiments utilized cells of passage 1, though cells were carried forward when possible.

ALI culture. Passaged bHNEs and bHBEs were grown at ALI as previously described $(11,28)$. In brief, passaged cell pellets were suspended in differentiation media (see Table 2 ) and counted with a hemocytometer. This mixture was seeded onto Transwell-Clear permeable supports $\left(0.33-\mathrm{cm}^{2}\right.$ filters, $0.4-\mu \mathrm{m}$ pore size; Corning Inc.) precoated with type IV collagen (Sigma-Aldrich) at approximately $260,000 \mathrm{cells} / \mathrm{cm}^{2}(80,000$ cells/insert). All cells were maintained in differentiation media, changing daily, removing apical media once confluent (approximately 3-4 days). Cells were maintained with basolateral media only, changing daily, for 4-5 weeks (depending on schedule) until testing. 
Table 3. Buffer solutions for Ussing studies

\begin{tabular}{lcc}
\hline Component & Basolateral chamber & Apical chamber \\
$\mathrm{NaCl}$ & $120 \mathrm{mM}$ & $1.2 \mathrm{mM}$ \\
$\mathrm{NaHCO}_{3}$ & $25 \mathrm{mM}$ & $25 \mathrm{mM}$ \\
$\mathrm{KH}_{2} \mathrm{PO}_{4}$ & $3.33 \mathrm{mM}$ & $3.33 \mathrm{mM}$ \\
$\mathrm{K}_{2} \mathrm{HPO}_{4}$ & $0.83 \mathrm{mM}$ & $0.83 \mathrm{mM}$ \\
$\mathrm{CaCl}_{2} \cdot 2 \mathrm{H}_{2} \mathrm{O}$ & $1.2 \mathrm{mM}$ & $1.2 \mathrm{mM}$ \\
$\mathrm{MgCl}_{2} \cdot 6 \mathrm{H}_{2} \mathrm{O}$ & $1.2 \mathrm{mM}$ & $1.2 \mathrm{mM}$ \\
$\mathrm{D}-$ glucose & $10 \mathrm{mM}$ & $10 \mathrm{mM}$ \\
$\mathrm{Na}$-gluconate & - & $141 \mathrm{mM}$
\end{tabular}

Ion transport. Once mature, select inserts were pretreated with VX-809 (3 $\mu$ M; Selleck Chemicals) for 48 hours prior to study. Inserts were removed from media and rinsed of any pretreatment drugs, then mounted in Ussing chambers (Physiologic Instruments) and studied as previously described (28). All studies were performed in an asymmetric chloride Ringer buffer (see Table 3 for buffer solutions), producing a basolateral-to-apical $\mathrm{Cl}^{-}$secretory gradient. Under voltage-clamp conditions, cells were treated apically with 100 $\mu \mathrm{M}$ amiloride to block ENaC and sodium transport. Forskolin $(10 \mu \mathrm{M})$ and IBMX $(100 \mu \mathrm{M})$ were added in both compartments to increase cAMP and stimulate CFTR. VX-770 $(1 \mu \mathrm{M})$ was added apically to potentiate CFTR. Finally, CFTR Inhibitor-172 $(10 \mu \mathrm{M})$ was added to the apical compartment to block CFTR currents. $I_{s c}$ and resistance were measured using Acquire and Analyze 2.3 software (Physiologic Instruments).

Whole-mount immunofluorescence. Inserts were washed in $1 \times$ PBS for 5 minutes 3 times, then fixed overnight in 4\% paraformaldehyde (Electron Microscopy Sciences) in PBS. Cells were permeabilized with $1 \%$ Triton X-100 (Sigma-Aldrich) in PBS for 15 minutes, then blocked with 2.5\% horse serum (Vector Laboratories) for 3 hours, all at room temperature. Inserts were washed in $1 \times$ PBS for 5 minutes 3 times, and immunostaining was performed with the antibodies and concentrations listed in Table 4 , using overnight $4^{\circ} \mathrm{C}$ incubation for primary antibodies and 1-hour room temperature incubation for secondary antibodies. Samples were washed with $1 \times$ PBS for 5 minutes 3 times, and the cells and permeable membranes were cut away from the insert with a razor. Membranes were mounted onto slides with ProLong Gold Antifade with DAPI (Thermo Fisher Scientific), and slides were imaged on a Nikon A1 inverted confocal microscope.

Statistics. Growth analyses include all center-specific bHNE and bHBE cultures from CF subjects over a 2-year period ( $n=102 \mathrm{bHNE}, 22 \mathrm{bHBE}$ samples), while all subsequent figures include only bHBE/bHNE culture pairs ( $n=11$ paired samples from 10 subjects). For $I_{s c}$ data, a minimum of 3 culture inserts were used to generate each data point; $n$ varied from 3 to 6 inserts per condition. All data were imported into Prism software (GraphPad Software) for analysis. Unpaired, 2-tailed $t$ tests (2 groups, with Holm-Šidák correction for multiple comparisons where applicable) or 2-way ANOVA (multiple groups, with Tukey's multiple comparison test where applicable) were used to compare continuous data, including growth characteristics and $I_{s c}$ with an a priori $\alpha(P)$ value less than 0.05 to determine statistical significance. Mean estimates $( \pm \mathrm{SEM})$ are presented for comparison of continuous data. Box-and-whisker plots represent minimum/ maximum (whiskers), 25th and 75th percentile (box limits), and median (central line). For all correlation

Table 4. Antibodies and fluorophores for immunofluorescence

\begin{tabular}{|c|c|}
\hline Primary antibody & Secondary antibody \\
\hline E-cadherin, Alexa Fluor 488-conjugated (eBioscience 53-3249-82) 1:500 & N/A \\
\hline Mouse acetylated $\alpha$-tubulin (Sigma-Aldrich T7451), 1:1,000 & Alexa Fluor 647-goat anti-mouse IgG2b (Life Technologies A21242), 1:500 \\
\hline Rabbit anti-CFTR (Sigma-Aldrich C7491), 1:100 & FITC-goat anti-Rabbit IgC (Invitrogen 656111), 1:500 \\
\hline Alexa Fluor 568 Phalloidin (Life Technologies A12380), 1:500 & $\mathrm{N} / \mathrm{A}$ \\
\hline
\end{tabular}


analyses, data from both samples from subject $\mathrm{J}$ were averaged to avoid unbalanced representation of the single subject. Correlation was determined by linear and nonlinear regression analysis as indicated; for linear regression, assumption of linearity was confirmed with multiple-runs tests. For all correlation analyses, agreement was tested through Bland-Altman analysis, plotting both the absolute difference between bHNE and bHBE measurements (bHNE - bHBE) and the relative difference (bHNE - bHBE)/(mean bHNE: $b H$ $\mathrm{BE}$ ) against the average of the $\mathrm{bHNE}$ and $\mathrm{bHBE}$ values (48). From each resulting data set, mean values, limits of agreement $( \pm 1.96 \mathrm{SD})$, and $95 \%$ CIs of the mean were calculated and plotted.

Study approval. All studies were approved by the Cincinnati Children's Hospital Medical Center Institutional Review Board. All subjects/caregivers provided written assent/consent prior to inclusion.

\section{Author contributions}

JJB and JPC conceived and designed the study. JJB, ETF, FJL, JDM, AJO, and LMS executed the study. JJB, ETF, FJL, JDM, AJO, LMS, and JPC analyzed and interpreted data. JJB and JPC drafted the manuscript for intellectual content.

\section{Acknowledgments}

The authors wish to thank Kristina Ray for her assistance in recruitment and records management and the Cincinnati Children's Hospital Cystic Fibrosis Center for their collaboration in this work. This work was supported by Cystic Fibrosis Foundation Therapeutics (CLANCY14XX0) and by the Cystic Fibrosis Foundation (CLANCY15R0).

Address correspondence to: John J. Brewington, 3333 Burnet Avenue/MLC 2021, Cincinnati Ohio, USA. Phone: 513.636.6771; Email: john.brewington@cchmc.org.

1. Patient Registry Annual Data Report. Cystic Fibrosis Foundation. https://www.cff.org/2014-Annual-Data-Report.pdf. Accessed June 7, 2018.

2. Rowe SM, Miller S, Sorscher EJ. Cystic fibrosis. N Engl J Med. 2005;352(19):1992-2001.

3. Darling RC, Disant'agnese PA, Perera GA, Andersen DH. Electrolyte abnormalities of the sweat in fibrocystic disease of the pancreas. Am J Med Sci. 1953;225(1):67-70.

4. Gibson LE, Cooke RE. A test for concentration of electrolytes in sweat in cystic fibrosis of the pancreas utilizing pilocarpine by iontophoresis. Pediatrics. 1959;23(3):545-549.

5. Welsh MJ. An apical-membrane chloride channel in human tracheal epithelium. Science. 1986;232(4758):1648-1650.

6. Boucher RC. Evidence for airway surface dehydration as the initiating event in CF airway disease. J Intern Med. 2007;261(1):5-16.

7. CFTR2 Database. Clinical and functional translation of CFTR. https://www.cftr2.org. Accessed June 4, 2018.

8. Van Goor F, et al. Correction of the F508del-CFTR protein processing defect in vitro by the investigational drug VX-809. Proc Natl Acad Sci USA. 2011;108(46):18843-18848.

9. Van Goor F, Yu H, Burton B, Hoffman BJ. Effect of ivacaftor on CFTR forms with missense mutations associated with defects in protein processing or function. J Cyst Fibros. 2014;13(1):29-36.

10. Lukacs GL, Verkman AS. CFTR: folding, misfolding and correcting the $\triangle \mathrm{F} 508$ conformational defect. Trends Mol Med. 2012;18(2):81-91.

11. Randell SH, Fulcher ML, O'Neal W, Olsen JC. Primary epithelial cell models for cystic fibrosis research. Methods Mol Biol. 2011;742:285-310.

12. Veit G, et al. From CFTR biology toward combinatorial pharmacotherapy: expanded classification of cystic fibrosis mutations. Mol Biol Cell. 2016;27(3):424-433.

13. Accurso FJ, et al. Effect of VX-770 in persons with cystic fibrosis and the G551D-CFTR mutation. N Engl J Med. 2010;363(21):1991-2003.

14. Wainwright CE, et al. Lumacaftor-ivacaftor in patients with cystic fibrosis homozygous for Phe508del CFTR. $N$ Engl $J$ Med. 2015;373(3):220-231

15. Davies JC, et al. Efficacy and safety of ivacaftor in patients aged 6 to 11 years with cystic fibrosis with a G551D mutation. Am J Respir Crit Care Med. 2013;187(11):1219-1225.

16. De Boeck K, et al. Efficacy and safety of ivacaftor in patients with cystic fibrosis and a non-G551D gating mutation. J Cyst Fibros. 2014;13(6):674-680.

17. Taylor-Cousar JL, et al. Tezacaftor-ivacaftor in patients with cystic fibrosis homozygous for Phe508del. N Engl J Med. 2017;377(21):2013-2023.

18. Rowe SM, et al. Tezacaftor-ivacaftor in residual-function heterozygotes with cystic fibrosis. N Engl J Med. 2017;377(21):2024-2035

19. Cutting GR. Cystic fibrosis genetics: from molecular understanding to clinical application. Nat Rev Genet. 2015;16(1):45-56.

20. Neuberger T, Burton B, Clark H, Van Goor F. Use of primary cultures of human bronchial epithelial cells isolated from cystic fibrosis patients for the pre-clinical testing of CFTR modulators. Methods Mol Biol. 2011;741:39-54.

21. Mou H, Brazauskas K, Rajagopal J. Personalized medicine for cystic fibrosis: establishing human model systems. Pediatr Pulmonol. 2015;50 Supp1 40:S14-S23. 
22. Worthington EN, Tarran R. Methods for ASL measurements and mucus transport rates in cell cultures. Methods Mol Biol. 2011;742:77-92.

23. Dekkers JF, et al. A functional CFTR assay using primary cystic fibrosis intestinal organoids. Nat Med. 2013;19(7):939-945.

24. Dekkers R, et al. A bioassay using intestinal organoids to measure CFTR modulators in human plasma. J Cyst Fibros. 2015;14(2):178-181.

25. Hug MJ, Tümmler B. Intestinal current measurements to diagnose cystic fibrosis. J Cyst Fibros. 2004;3 Suppl 2:157-158.

26. van Barneveld A, Stanke F, Ballmann M, Naim HY, Tümmler B. Ex vivo biochemical analysis of CFTR in human rectal biopsies. Biochim Biophys Acta. 2006;1762(4):393-397.

27. Ahmadi S, et al. Phenotypic profiling of CFTR modulators in patient-derived respiratory epithelia. NPJ Genom Med. $2017 ; 2: 12$.

28. Brewington JJ, et al. Detection of CFTR function and modulation in primary human nasal cell spheroids. J Cyst Fibros. 2018;17(1):26-33.

29. Pranke IM, et al. Correction of CFTR function in nasal epithelial cells from cystic fibrosis patients predicts improvement of respiratory function by CFTR modulators. Sci Rep. 2017;7(1):7375.

30. Guimbellot JS, et al. Nasospheroids permit measurements of CFTR-dependent fluid transport. JCI Insight. 2017;2(22):e95734

31. Knowles MR, Carson JL, Collier AM, Gatzy JT, Boucher RC. Measurements of nasal transepithelial electric potential differences in normal human subjects in vivo. Am Rev Respir Dis. 1981;124(4):484-490.

32. Pedersen PS, Frederiksen O, Holstein-Rathlou NH, Larsen PL, Qvortrup K. Ion transport in epithelial spheroids derived from human airway cells. Am J Physiol. 1999;276(1 Pt 1):L75-L80.

33. Mosler K, et al. Feasibility of nasal epithelial brushing for the study of airway epithelial functions in CF infants. J Cyst Fibros. 2008;7(1):44-53.

34. Liu X, et al. ROCK inhibitor and feeder cells induce the conditional reprogramming of epithelial cells. Am J Pathol. 2012;180(2):599-607.

35. Reynolds SD, et al. Airway progenitor clone formation is enhanced by Y-27632-dependent changes in the transcriptome. Am $J$ Respir Cell Mol Biol. 2016;55(3):323-336.

36. Schögler A, et al. Characterization of pediatric cystic fibrosis airway epithelial cell cultures at the air-liquid interface obtained by non-invasive nasal cytology brush sampling. Respir Res. 2017;18(1):215.

37. Bishop MD, et al. The cystic fibrosis transmembrane conductance regulator gene and ion channel function in patients with idiopathic pancreatitis. Hum Genet. 2005;118(3-4):372-381.

38. Wine JJ, et al. In vivo readout of CFTR function: ratiometric measurement of CFTR-dependent secretion by individual, identifiable human sweat glands. PLoS ONE. 2013;8(10):e77114.

39. Moss RB, et al. Efficacy and safety of ivacaftor in patients with cystic fibrosis who have an Arg117His-CFTR mutation: a double-blind, randomised controlled trial. Lancet Respir Med. 2015;3(7):524-533.

40. Ratjen F, et al. Efficacy and safety of lumacaftor and ivacaftor in patients aged 6-11 years with cystic fibrosis homozygous for F508del-CFTR: a randomised, placebo-controlled phase 3 trial. Lancet Respir Med. 2017;5(7):557-567.

41. Devalia JL, Davies RJ. Human nasal and bronchial epithelial cells in culture: an overview of their characteristics and function. Allergy Proc. 1991;12(2):71-79.

42. Devalia JL, Sapsford RJ, Wells CW, Richman P, Davies RJ. Culture and comparison of human bronchial and nasal epithelial cells in vitro. Respir Med. 1990;84(4):303-312.

43. Lopez-Souza N, Avila PC, Widdicombe JH. Polarized cultures of human airway epithelium from nasal scrapings and bronchial brushings. In Vitro Cell Dev Biol Anim. 2003;39(7):266-269.

44. Alves MP, et al. Comparison of innate immune responses towards rhinovirus infection of primary nasal and bronchial epithelial cells. Respirology. 2016;21(2):304-312.

45. Thavagnanam S, Parker JC, McBrien ME, Skibinski G, Shields MD, Heaney LG. Nasal epithelial cells can act as a physiological surrogate for paediatric asthma studies. PLoS ONE. 2014;9(1):e85802.

46. Ogilvie V, et al. Differential global gene expression in cystic fibrosis nasal and bronchial epithelium. Genomics. 2011;98(5):327-336.

47. Gentzsch M, et al. Pharmacological rescue of conditionally reprogrammed cystic fibrosis bronchial epithelial cells. Am J Respir Cell Mol Biol. 2017;56(5):568-574.

48. Giavarina D. Understanding Bland Altman analysis. Biochem Med (Zagreb). 2015;25(2):141-151. 